\title{
Non-linear analysis of geomagnetic time series from Etna volcano
}

\author{
G. Currenti ${ }^{1}$, C. Del Negro ${ }^{1}$, L. Fortuna ${ }^{2}$, R. Napoli ${ }^{1}$, and A. Vicari ${ }^{1,2}$ \\ ${ }^{1}$ Istituto Nazionale di Geofisica e Vulcanologia - Sezione di Catania, Italy \\ ${ }^{2}$ Dipartimento di Ingegneria Elettrica, Elettronica e dei Sistemi - Università di Catania, Italy
}

Received: 26 June 2003 - Revised: 13 October 2003 - Accepted: 12 November 2003 - Published: 25 February 2004

\begin{abstract}
An intensive nonlinear analysis of geomagnetic time series from the magnetic network on Etna volcano was carried out to investigate the dynamical behavior of magnetic anomalies in volcanic areas. The short-term predictability of the geomagnetic time series was evaluated to establish a possible low-dimensional deterministic dynamics. We estimated the predictive ability of both a nonlinear forecasting technique and a global autoregressive model by comparing the prediction errors. Our findings highlight that volcanomagnetic signals are the result of complex processes that cannot easily be predicted. There is slight evidence based on nonlinear predictions, that the geomagnetic time series are to be governed by many variables, whose time evolution could be better regarded as arising from complex high dimensional processes.
\end{abstract}

\section{Introduction}

The geomagnetic field as a whole varies in time and space, but in general the time variations change only slightly with position and the space changes have very little time dependence. The major space changes derive from the local field (attributable to static crustal sources), which does not change with time, while time changes come about either from the Earth's core (slow secular variation) or from ionospheric and magnetospheric current systems (diurnal solar variation and magnetic storms) (Barraclough and De Santis, 1997; Hongre et al., 1999). The most spectacular exceptions to this rule have been observed close to eruptive events, when there may be changes in the local field due to some time change in physical parameters (e.g. temperature and stress) of the volcanic rocks that affect the magnetization.

For all practical purposes, volcanomagnetic monitoring is concerned with detection of local field changes attributable to the dynamics of a volcano and removal of the geomagnetic

Correspondence to: C. Del Negro

(delnegro@ct.ingv.it) field variations with no geophysical significance. In general, the spatial distribution of the secular variation and external field fluctuations can be considered uniform (because of the large distance of sources), and therefore simple differences in the total intensity with respect to the simultaneous value at a remote reference (usually located at a magnetically quiet site) are used to reduce these effects. Differentiating these data reduces the magnetic disturbance by about $95 \%$ (Davis et al., 1981) and the average of these differentiated data on a daily basis reduces the standard deviations by a factor of 10 (Mueller and Johnston, 1998). Normally, measurement accuracy for these data is better than $1 \mathrm{nT}$, depending on site separation and local magnetic gradients. After processing, these total intensity data are routinely searched for any time changes. Unfortunately, volcano magnetic signals are very small and must be detected in the presence of considerable noise. Very much larger time variations, with periods from minutes to years, are present in these differences. For example, also in the absence of any volcanic activity, some of the time changes can occur because of secondary fields induced in the crustal rocks by external current systems. Where the electrical conductivity of the rocks of a volcano changes over short distances, time changes can vary correspondingly. Differences in underground conductivity between two close (order of $10 \mathrm{~km}$ apart) sites will make the time variable fields different, even if the primary field from the magnetosphere is the same (Parkinson, 1983). On the other hand, variable induced magnetization, due to large susceptibility contrasts, could locally modify a magnetic disturbance field by an amount up to $5 \mathrm{nT}$ (Davis et al., 1979).

These effects can seriously hinder the accurate detection of volcanomagnetic signals. When we expect only a small volcanomagnetic signal, due either to a weak volcanic activity or to the large distance between the source zone and the observation sites, the presence of non-volcanic changes not only makes signal detection more difficult but may also lead us to misinterpret data. With the improvement in data acquisition systems and the application of noise reduction procedures, significative magnetic field anomalies have been 
detected during main phases of volcanic activity (Del Negro and Currenti, 2003), even if many problems still remain to be resolved for understanding the temporal dynamics of the local magnetic anomalies. The dependence on time of volcanomagnetic signals is not yet completely understood, therefore a robust analysis, able to describe the dynamics of the processes, could be useful to improve the comprehension of anomalies.

To investigate the temporal dynamics of geomagnetic field in active volcanic areas, we analyzed data collected from the magnetic monitoring network at Etna volcano (Del Negro et al., 2002). We have been using methods based on the theory of nonlinear dynamical systems to explore if there is evidence of a possible low-dimensional nonlinear behavior of the system. The reliability and accuracy of forecasting models were evaluated to figure out the behavior of geomagnetic activity, and to examine the dynamical features of the system responsible for the magnetic anomalies. The forecasting models could represent a valuable tool to study the short-term predictability of volcanomagnetic processes. Two different autoregressive models are compared: the one is a global linear autoregressive model and the other is a nonlinear forecasting model based on local autoregressive techniques. The quality of the nonlinear predictions is evaluated by appraising the accuracy of the forecasting with respect to linear predictions (Cuomo et al., 1999). By comparing the forecasting errors computed for both models, it is possible to discern which model is more reliable for describing the dynamical behavior of the observed magnetic variations. The global linear model starts out from the assumption that the signal is generated by a stochastic process, whereas the local linear one assumes that the underlying physical mechanism is low dimensional and deterministic. The local linear prediction is one of the several methods that are suggested as a test for discerning chaotic behavior from measured data (Casdagli, 1991; Farmer and Sidorowich, 1987; Tsonis et al., 1994). Therefore this analysis permits to evaluate two probable dynamical behaviors and distinguish randomness from chaos. If the best forecasting occurs using the global model, the data are well described by a linear stochastic process, otherwise it is more consistent to support the idea of the existence of a nonlinear deterministic motion. In this way it will be possible to understand which are the dominant features of the dynamical process that leads the time-dependent magnetic anomalies.

\section{Methods of analysis}

We use the nonlinear prediction method of Tsonis and Elsener (1992) to investigate the dynamical behavior of geomagnetic time series. This method employs a local linear forecasting to identify chaotic behavior in natural time series. The idea behind using prediction as evidence of chaos is very intuitive. The limited predictive ability of a chaotic dynamical system is due to its sensitivity to initial conditions. Therefore, it is expected that chaos is to be characterized by a de- crease in the correlation between predicted and observed values as prediction time increases. If the system is chaotic, then the decrease of predictive ability with prediction time gives an estimate of the Lyapunov exponent. It is worth noting that the average exponential rate at which initial trajectories diverge depends on its Lyapunov exponent. This property can be used to distinguish chaotic behavior from randomness. In fact, for a random signal, it is expected that the forecasting property doesn't depend on the prediction interval. On the other hand, because of the sensitivity to initial conditions, long-term forecasting is not possible for a chaotic system and short-term prediction could be suitable using nonlinear prediction. The forecasting approach is based on predicting the future value of a data point by considering the time evolution of its neighbors in the reconstructed phase space. The algorithm is based on three fundamental steps: (i) building the embedding state, (ii) searching the nearest neighbors at each point, and (iii) computing the local model, relying on only the neighboring points, to generate the predictions.

The first step is the choice of an appropriate time delay and an embedding dimension in order to construct the delay vectors, using the embedding theorem by Takens (1981). Given a time series $x(t)$, the reconstructed delay vector is represented by:

$$
\begin{gathered}
\boldsymbol{X}\left(t_{n}\right)=\left(x\left(t_{n}\right), x\left(t_{n}-\tau\right), x\left(t_{n}-2 \tau\right), \ldots\right. \\
\left.\ldots, x\left(t_{n}-(E-1) \tau\right)\right)
\end{gathered}
$$

where $\tau$ is the time delay and $E$ is the embedding dimension.

The idea behind using prediction techniques to detect chaotic behavior is supported by the nonlinear systems theory. In fact, it is well known that if a deterministic dynamics exists, it is possible to determine the location of the next point in the reconstructed phase space by a map $f$ such as:

$$
\boldsymbol{X}_{t+1}=f\left(\boldsymbol{X}_{t}, \boldsymbol{X}_{t-1}, \ldots, \boldsymbol{X}_{t-E+1}\right)
$$

The quality of forecasting basically depends on the capability to approximate the dynamics $f$. Regarding this, the dynamics can be analyzed in the reconstructed phase space by the delay vectors. The procedure to reconstruct the orbits in the pseudo-phase space is associated with the choice of the embedding dimension and the time delay (Abarbanel, 1995). The embedding theorem is suitable in principle for any value of $\tau$. From a mathematical point of view, we could arbitrarily choose $\tau$ value, since it is assumed that the data have infinite precision. But in applications with real data this is never going to happen and the choice of time delay is not trivial. Unfortunately, a rigorous method to determine its optimal value doesn't exist. If the time delay is too short, the elements of the delay vectors will not be independent enough. On the other hand, if $\tau$ is too large the coordinates may be uncorrelated and significant dynamical information could be lost.

The second step, in order to predict a time $L$ ahead of $t_{n}$, is to select a neighborhood around the point $\boldsymbol{X}\left(t_{n}\right)$ in the reconstructed phase space. Because of the assumption of the deterministic behavior, it is reasonably expected that the evolution of the selected vector is correlated with the evolution of 


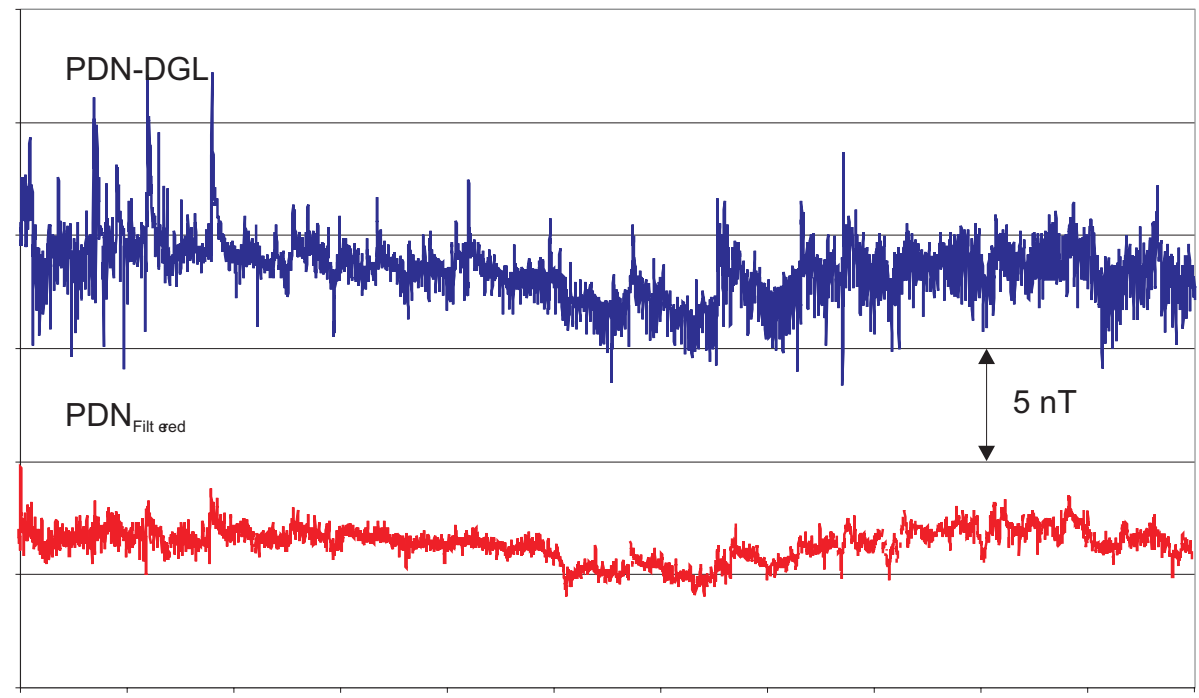

Oct-01 Nov-01 Dec-01 Jan-02 Feb-02 Mar-02 Apr-02 May-02 Jun-02 Jul-02 Aug-02
Fig. 1. Hourly mean differences of total magnetic intensity of PDN with respect to DGL from October 2001 to August 2002 (blue) and cleaned differences field after multichannel filtering (red). the neighboring vectors, which could provide predictions of the future value of $\boldsymbol{X}\left(t_{n}\right)$ through an appropriate local model. Given the embedding vector, we select the neighboring vectors closer to $\boldsymbol{X}\left(t_{n}\right)$, using a distance function. For computing the distance in the reconstructed phase space, we calculate the Euclidean norm between the point $\boldsymbol{X}\left(t_{n}\right)$ and all the remaining points in the neighborhood. For each point all past points in the reconstructed phase space are considered, they are tested for closeness and most of them are rejected because they are too far away.

The third step evaluates a suitable local mapping that describes how all points belonging to the neighborhood advance to future time $t_{n+L}$. Generally local averaging models and local linear models are used. The methods based on local averaging models are particularly useful when the data sets are sparse, because they do not require a large number of points (Sugihara and May, 1990). On the other hand, they are less accurate than the local linear models (Kantz and Schreiber, 1997). Since a sufficiently large number of data is available, we prefer to employ a local linear approach defined by:

$x\left(t_{n}+L\right)=\boldsymbol{A} \boldsymbol{X}\left(t_{n}\right)$

where $L$ is the prediction interval, $\boldsymbol{X}\left(t_{n}\right)$ is the delay vectors and $\boldsymbol{A}$ the parameters vector. To determine these parameters in the model, the $K$ nearest neighbors are selected and the linear approximation is solved for each point of the time series, because the model is valid only locally. To this end, the search of neighborhood must be repeated for each point in the phase space to determine the local model, associated to that point. Since the local model, computed at each step, might not always be the same, the whole procedure is nonlinear. To estimate the prediction for the $L$ step ahead, the forecasting error is computed $e(L)=x(n+L)-x^{\prime}(n+L)$. The normalized mean square error (NMSE) between predictors and real data is used to quantify the predictive ability. The $\operatorname{NMSE}(L)$ of the sequence $\boldsymbol{X}$, is defined by:

$\operatorname{NMSE}(L)=\sqrt{\frac{\sum_{i=1}^{N} e_{i}^{2}(L)}{N \sigma_{Y}^{2}}}$

where $\sigma$ denotes the standard deviation of the $\boldsymbol{X}$ time series and $N$ the length of data. The rapid increase of the NMSE in function of prediction time interval $L$ is a strong signature of chaos (Tsonis and Elsener, 1992) since it is influenced by the chaotic divergence of initially close trajectory. The prediction error is affected by critical selection of the algorithm parameters, such as the embedding dimension $E$, the delay time $\tau$, the neighborhood size $K$. We took care on the choice of the optimal value of these parameters. Particularly, we analyzed how the NMSE, representing the quality index of the model, changes by varying the value of these parameters, which provide some relevant dynamical properties of the system.

\section{Observed data}

Over the last two decades, the Laboratory of Geomagnetism of INGV-CT has been intensively monitoring the magnetic field on Etna volcano. We have observed significant correlations between volcanic activity and changes in the local magnetic field, up to a few tens of nanoteslas (e.g. Del Negro et al., 1997; Del Negro and Ferrucci, 1998; Del Negro and Currenti, 2003). Detection of clear magnetic signals associated with the renewal of the volcanic activity led to an increase in the magnetic monitoring of Etna. Since the end of 1998 a permanent magnetic network has been set up (Del Negro et al., 2002). All remote stations are equipped with Overhauser effect magnetometers (0.01 nT sensitivity) and synchronously sample the Earth's magnetic field every $10 \mathrm{~s}$. 


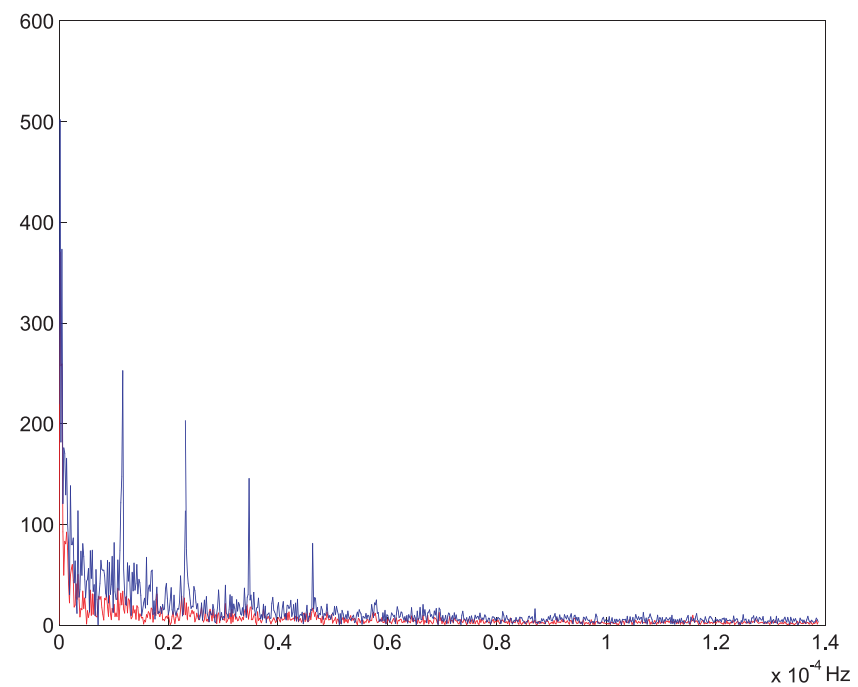

Fig. 2. Power spectra of the simple differences (blue) and cleaned differences (red) between PDN and DGL.

All the work has been based on data collected from October 2001 to August 2002 at two selected stations: DGL and PDN, which are about $1 \mathrm{~km}$ far away. Before anything else, we have used total field differences between two adjacent stations because they have the advantage that noise spikes are easily detected and edited out. In the second place, the hourly mean data were computed (Fig. 1) and analyzed to avoid as much as possible contamination from external magnetic fields. Reduction of these disturbances by taking simple differences between total field readings at space stations is unsatisfactory, because total field differences are dependent on the direction of the disturbing field (Davis et al., 1979). It is known that improvement is possible when three component observatory data are available from a station in the vicinity, but not necessarily immediate, of the total field array (Davis et al., 1981). We identified and removed the variations of ionosphere and magnetosphere origin by multichannel filtering method (Davis and Johnston, 1983) using L'Aquila Geomagnetic Observatory component fields as input to the filter. This method involves finding the multichannel filters that estimate the field variation at PDN station from the field of the DGL station and a three-component magnetometer. The difference is then taken between total field and estimated field, leaving a cleaned total field. The cleaned differences field shows that the filtering process is gainful during both disturbed and quite days (Fig. 1).

In Fig. 2 are shown the power spectra of simple differences and cleaned differences between DGL and PDN stations. The analysis of the simple differences power spectra clearly shows prominent peaks centered around diurnal components at the period of 8,12 and $24 \mathrm{~h}$. Although these variations are the most regular of the all geomagnetic changes, they tend not to repeat exactly itself but relevant differences could be noted from day to day (Okeke and Hamano, 2000). The dayto-day variability is thought to arise from the variations of

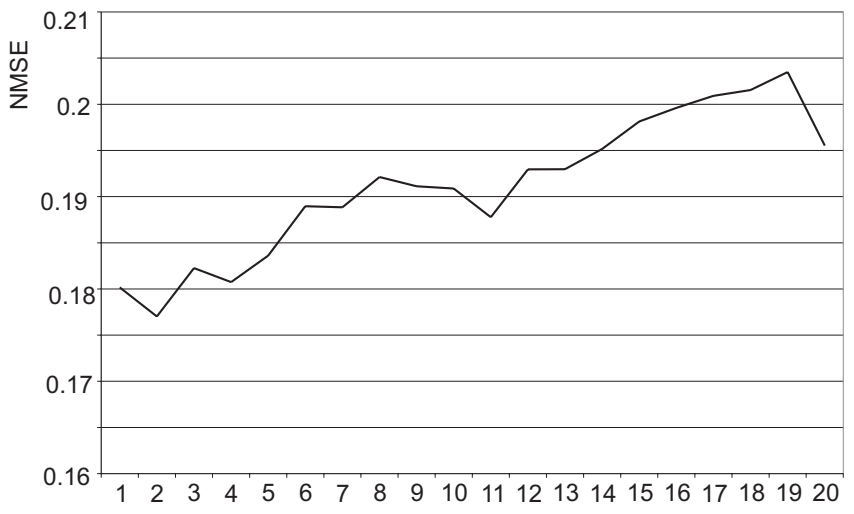

Fig. 3. NMSE versus the time delay.

the solar and geomagnetic activity. These diurnal magnetic variations are filtered out in the cleaned differences power spectra. After having removed the dominant periodic components, the power spectra of filtered differences appears to be aperiodic and broad band. The spectrum does not show any privileged frequencies, but rather a linear decay which links the whole range of frequency domain (Fig. 2). These characteristics are consistent with the behavior of almost all nonlinear dynamical systems or chaotic ones (Kostelich, 1997). The broad band spectrum claims that a possible nonlinear deterministic component could be present in cleaned differences field. Nevertheless, it is worth noting that a broad band spectrum can correspond either to a low dimensional chaotic system or a stochastic time series. An obvious distinction between deterministic and stochastic processes is not so simple. Therefore, we look at the nonlinear prediction approaches as a way to provide evidences on the mechanism generating the time dependent variations.

\section{Predictions}

Before computing the local model over cleaned differences field, we firstly have to face the proper choice of the time delay and the embedding dimension. As described by Sugihara and May (1990), the local model can suggest by itself the best values for the embedding. In fact, once the suitable embedding is reached, the predictability remains unchanged even if the $E$ and $\tau$ values are increased. For a prediction time interval of $L=1$, we estimate the prediction improvement for different values of $E$ and $\tau$. When the nearest neighbors in the reconstructed phase space are searched, one should avoid using neighbors in phase space that lie on the same trajectory segment, because they contain only little information about the underlying deterministic dynamics. Delay vectors, which are close in the phase space, are also close in time because of continuous time evolution. Therefore, in the local linear method, points close in time are to be rejected. In practice, to prevent a temporal correlation, we divide the time series into two parts: a learning data set $X_{1}$ and a testing data set $X_{2}$. The first part of the series $X_{1}$ is used to find the 


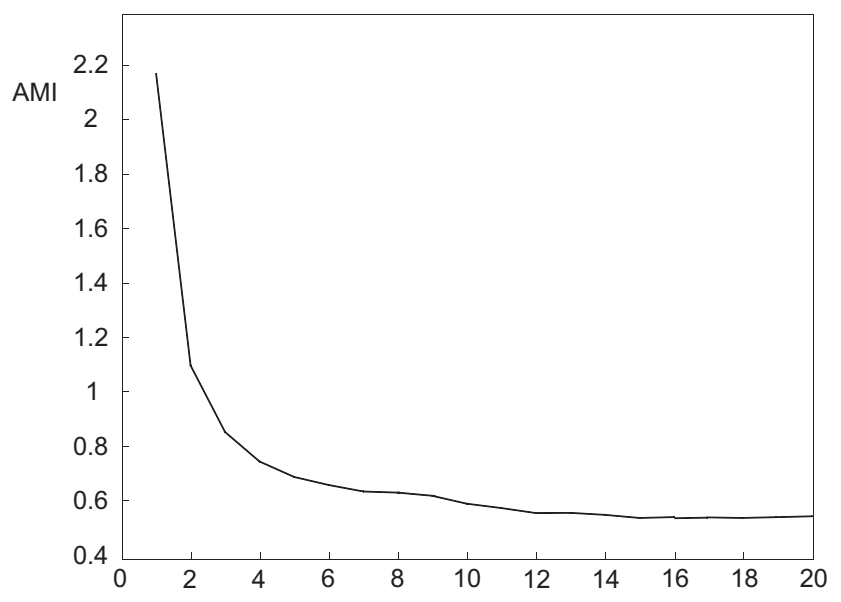

Fig. 4. Determination of $\tau$ using average mutual information (AMI) criterion.

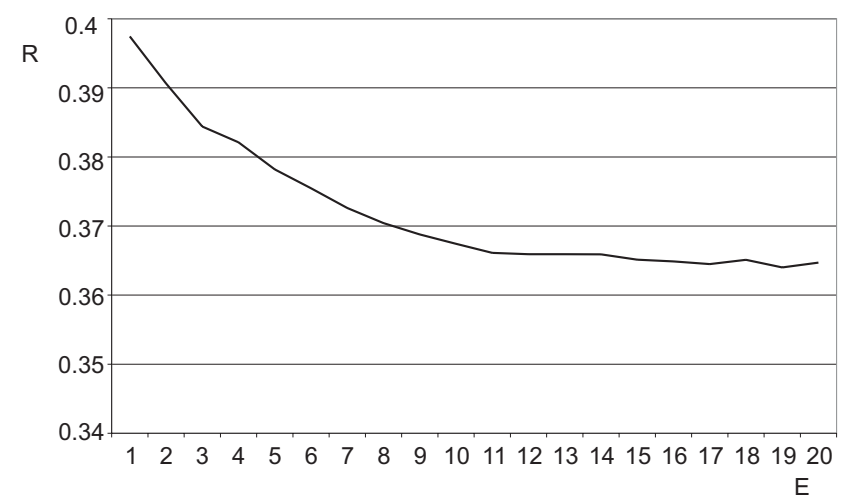

Fig. 5. $R$ coefficient as a function of the embedding dimension $E$.

closest neighbors of a point in $X_{2}$, on which the forecast is performed and the NMSE is computed.

Having fixed the neighbor size $K$ at $50 \%$ of available data, we firstly look for the better value of $\tau$. The quality of prediction is evaluated for different values of $\tau$ and $E$. For each $\tau$, an average of the NMSE over $E$ is computed and the results are shown in Fig. 3. The predictions seem to be slightly sensitive to the choice of $\tau$, even if they are more precise for lower $\tau$. A general method for the choice of the time delay is based on the search for the first relative minimum of the average mutual information (AMI) (Abarbanel, 1995). However, the AMI function, computed over our data set, just gradually declines without any clearly detectable minima (Fig. 4). In such a case, the advice of Abarbanel et al. (1993) is to take $\tau=1$. Under these circumstances an exact choice of time delay is not deemed to be critical and, after choosing a time delay of $\tau=1$, we test the ability of the local prediction as a function of $E$. The optimal value of the embedding dimension is evaluated by computing the minimum of the following $R$ coefficient:

$R\left(E_{i}\right)=\left\langle\operatorname{NMSE}\left(L, E_{i}\right)\right\rangle$

where $L=1 \ldots 20$ and $E_{i}=1 \ldots 20$.

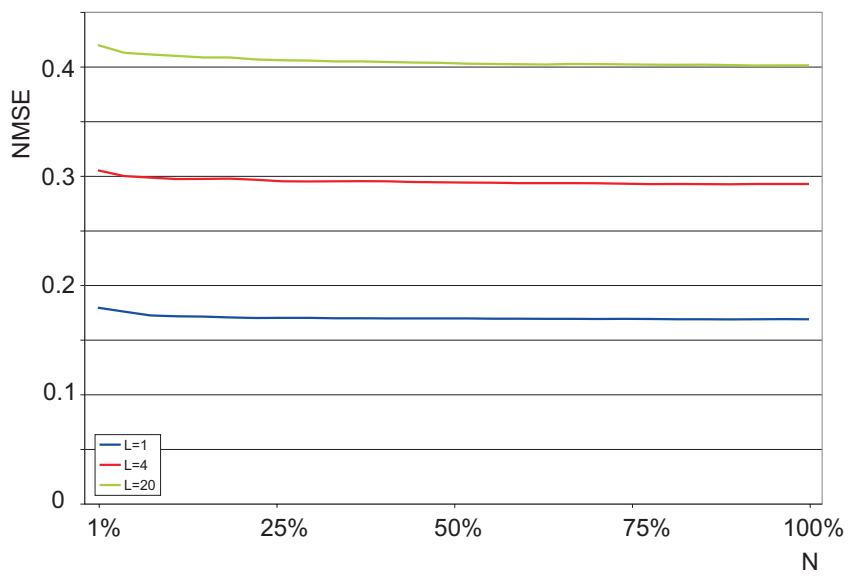

Fig. 6. The normalized mean square error (NMSE) for different values of prediction time interval $(L=1, \ldots, 20)$ vs. neighbor size $K$.

An improvement is obtained when $E$ increases, even if the $R$ doesn't go below 0.36 (Fig. 5). The optimum embedding dimension seems to be about 10 , as the $R$ coefficient does not improve for higher values. The statistical properties of the local model also depend on the neighborhood size that leads the locality of the approximation. The behavior of NMSE is analyzed with respect to the number of neighbors $K$. In this regard, $K$ works as a smoothing parameter. The smaller $K$ is, the more local the approximation is. On the other hand, the neighborhood size should be large enough to compute a statistically valid local model. Moreover, the smallest distance between the reference point and its neighbors has to be greater than the noise amplitude; otherwise the possible deterministic properties of the system are hidden. In our case, we evaluate the NMSE for $K$ number bigger than $1 \%$ of the learning data set. For different $L$ values the trend of NMSE in function of the number of the nearest neighbors is shown in Fig. 6. The NMSE always grows exponentially and reaches rapidly its highest value. It remains unchanged for $K$ bigger than $25 \%$.

After the best parameter of the local model have been chosen, we compare the local linear model with respect to a global linear model having the same $E$ and $\tau$ parameters, and we look for difference between the local and global properties. The NMSE is evaluated for the global model and the local one as the time prediction $L$ increases (Fig. 7). It is worth noting that the prediction error strongly depends on prediction time interval, revealing the unpredictability of future values for long time steps.

\section{Conclusions}

We have analyzed geomagnetic time series (lasting about one year) from Etna volcano, using the local prediction and the global linear methods. Our aim is to determine if there is evidence for understanding and describing the temporal dynamics of volcanomagnetic signals. A common problem in 


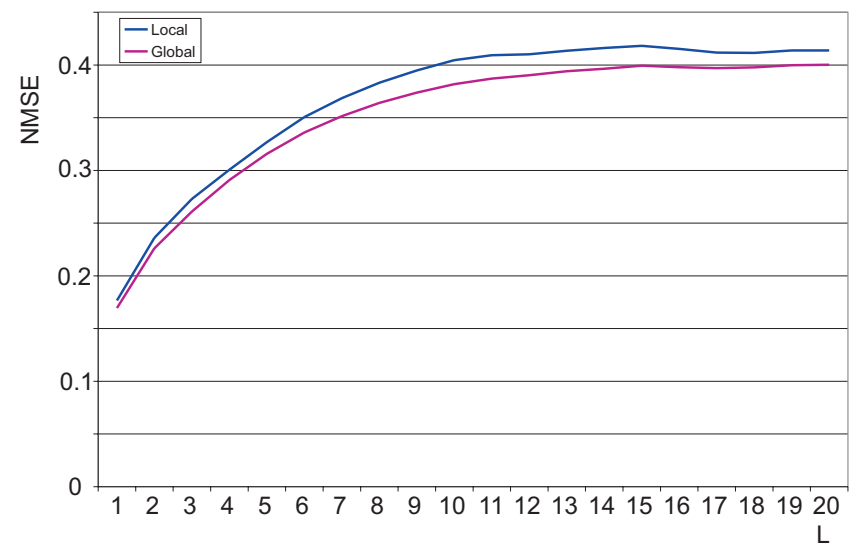

Fig. 7. The normalized mean square error (NMSE) for global (red) and local(blue) approach as time prediction increases up to $L=20$.

the study of aperiodic and irregular signals is to establish whether the underlying process is governed by low dimensional deterministic dynamics or by some non-deterministic rules, which cannot be described with finite numbers of state variables. Conventional spectral analysis is not able to distinguish chaos from random signals, since in both cases the signals have continuous broad band power spectra. So the prediction ability was evaluated.

Applying the local forecasting algorithm on the magnetic data, the NMSE prediction error does not exhibit a significant minimum for any $K$ value less than the samples number. Increasing the number of neighbors $K$ used for estimating the model, the local linear prediction becomes a global linear one. Best predictions are achieved for the largest $K$, highlighting that the global autoregressive model seems to be superior. A rapid increase of NMSE is observed as the time prediction interval increases, for both global and local prediction models. However, comparing the predictive ability of the two forecasting approaches, the global model is always better than the local one. Since the time series is better modeled by a linear stochastic autoregressive process, it claims that the degrees of freedom of the system are not few. Moreover, in agreement with the hypothesis of high dimensionality of the system, the quality of predictions increases with the number of neighbors used to compute the model.

These results could have important implications on the study of the dynamical behavior of the volcanomagnetic signals. They underline that volcanomagnetic signals are the result of complex processes that cannot be easily predicted. The geomagnetic time series seem to be governed by many variables whose time evolution could be better regarded as arising from stochastic processes. The application of nonlinear forecasting techniques has not provided strong evidence of nonlinear deterministic dynamics in volcanomagnetic data.

Acknowledgements. We are indebted to all personal of Geomagnetism Laboratory of INGV-CT who guarantee the regular working of the permanent magnetic network on Etna volcano. The manuscript gained in clarity thanks to constructive criticisms by
L. Chua and A. De Santis. This research was carried out in the frame of the TecnoLab, the laboratory for technological advance in geophysics organized by DIEES-UNICT and INGV-CT, and benefited of support by project EPOT of the Gruppo Nazionale per la Vulcanologia.

Edited by: R. Grimshaw

Reviewed by: two referees

\section{References}

Abarbanel, H. D. I.: Analysis of observed chaotic data, Springer Verlag, 1995.

Abarbanel, H., Brown, R., Sidorowich, J., Tsimring, L.: The analysis of observed Chaotic data in physical systems, Reviews of Modern Physics, 65, 1331-1392, 1993.

Barraclough, D. R. and De Santis, A.: Some possible evidence for a chaotic geomagnetic field from observational data, Phys. Earth Planet. Inter., 99, 207-220, 1997.

Casdagli, M.: Chaos and deterministic versus stochastic nonlinear modeling, J. Roy. Stat. Soc., 54, 303-328, 1991.

Cuomo, V., Lapenna, P., Macchiato, M., Serio, C., and Telasca, L.: Stochastic behoviour and scaling laws in geoelectrical signals measured in a seismic area of southern Italy, Geophys. J. Int., Vol 139, 889-894, 1999.

Currenti, G., Del Negro, C., Fortuna, L., Vicari, A.: Nonlinear identification of complex geomagnetic models: an innovative approach, Nonlinear Phenomena in Complex Systems, 6:1, 524533, 2003.

Davis, P. M., Stacey, F. D., Zablocki, C. J., and Olson, J. V.: Improved signal discrimination in tectonomagnetism: discovery of a volcanomagnetic effect at Kilauea, Hawaii, Phys. Earth Planet. Inter., 19, 331-336, 1979.

Davis, P. M., Jackson, D. D., Searls, C. A., and McPherron, R. L.: Detection of tectonomagnetic events using multichannel predictive filtering, J. Geophys. Res., 86, 1731-1737, 1981.

Davis, P. M. and Johnston, M. J.: Localized geomagnetic field changes near active faults in California 1974-1980, J. Geophys. Res., 88, 9452-9460, 1983.

Del Negro, C. and Currenti, G.: Volcanomagnetic signal associated with the 2001 flank eruption of Mt. Etna, Geophys. Res. Lett., 30, 7, 2003

Del Negro, C., Ferrucci, F., and Napoli, R.: The Permanent Network for Magnetic Surveillance of Mt. Etna: Changes in the Geomagnetic Total Intensity Observed in 1995, Acta Vulcanologica, 9, 1-7, 1997.

Del Negro, C. and Ferrucci, F.: Magnetic history of a dyke on Mt. Etna, Geophys. J. Int., 133, 451-458, 1998.

Del Negro, C., Napoli, R., and Sicali, A.: Automated system for magnetic monitoring of active volcanoes, Bull. Volcanol., 64, 94-99, 2002.

Farmer, J. D. and Sidorowich, J. J.: Predicting chaotic time series, Phys. Rev. Lett., 59, 845, 1987.

George, B., Renuka, G., Satheesh Kumar, K., Anil Kumar, C. P., and Venugopal, C.: Nonlinear time series analysis of the fluctuations of the geomagnetic horizontal field, Ann. Geophysicae, 20, 175183, 2001.

Hongre, L., Sailhac, P., Alexandrescu, M., andDubois, J.: Nonlinear and multifractal approaches of geomagnetic field, Phys. Earth Planet. Inter., 110, 157-190, 1999. 
Kantz, H. and Schreiber, Th.: Nonlinear Time Series Analysis, Cambridge Univ. Press, Cambridge, 1997.

Kostelich, E. J.: The analysis of chaotic time-series data, Systems and Control Letters, 31 313-319, 1997.

Mueller, R. J. and Johnston, M. J. S.: Review of magnetic field monitoring near active faults and volcanic calderas in California: 1974-1995, Phys. Earth Planet. Inter., 105, 131-144, 1998.

Okeke, F. N. and Hamano, Y.: Daily variations of geomagnetic H D and Z-field at equatorial latitudes, Earth Planets Space, 52, 237243, 2000.

Parkinson, W. D.: Introduction to geomagnetism, Scottish Academic Press, 1983.

Price, C. P., Prickard, D., and Bischoff, J. E.: Nonlinear input/outputof the auroral electrojet index, J. Geophys. Res., 99, $13227-13238,1994$.
Sugihara, G. and May, R. M.: Nonlinear forecasting as a way of distinguishing chaos from measurement error in time series, Nature, 344, 734-741, 1990.

Takens, F.: Detecting strange attractors in turbulence, Springer Lec. Notes in Math., Springer-Verlag, New York, 898, 366-381, 1981.

Tsonis, A. A. and Elsner, J. B.: Nonlinear prediction as a way of distinguishing chaos from random fractal sequences, Nature, 358, 217-220, 1992.

Tsonis, A. A., Triantafyllou, G. N., and Elsner, J. B.: Searching for determinism in observed data: a review of the issue involved, Nonlin. Proc. Geophys, 1, 12-25, 1994. 